\title{
Programme overview
}

\begin{tabular}{|c|c|c|c|}
\hline Mednet & 2000 & DAY 1 & Thursday Nov. 23rd \\
\hline & Tutorial1 & Tutorial 2 & Tutorial 3 \\
\hline & Room: Aud. 6 & Room: Aud. 2 & Room: computer class \\
\hline & $\mathrm{T} 1$ & $\begin{array}{r}\mathrm{T} 2 \\
\end{array}$ & T3 \\
\hline $\begin{array}{l}9 \mathrm{9h00-9h20} \\
9 \mathrm{~h} 20-9 \mathrm{~h} 40 \\
9 \mathrm{~h} 40-10 \mathrm{~h} \\
10 \mathrm{~h} 00-10 \mathrm{~h} 20 \\
10 \mathrm{~h} 20-10 \mathrm{~h} 40 \\
\end{array}$ & $\begin{array}{l}\text { Collecting and calcu- } \\
\text { lating medical data } \\
\text { via the Internet } \\
\text { Elsner }\end{array}$ & $\begin{array}{l}\text { How to create dynamic } \\
\text { interactive medical web } \\
\text { applications } \\
\text { Pretlow }\end{array}$ & $\begin{array}{l}\text { Qualité de l'infor- } \\
\text { mation médicale } \\
\text { sur Internet } \\
\text { Jamoulle }\end{array}$ \\
\hline 10h40-11h & Coffee & & \\
\hline $\begin{array}{l}\frac{11 \mathrm{~h} 00-11 \mathrm{~h} 20}{11 \mathrm{~h} 20-11 \mathrm{~h} 40} \\
\frac{11 \mathrm{~h} 40-12 \mathrm{~h}}{12 \mathrm{~h} 00-12 \mathrm{~h} 20} \\
\end{array}$ & & & \\
\hline $12 \mathrm{~h} 20-14 \mathrm{~h}$ & LUNCH & & \\
\hline & $\begin{array}{r}\text { Tutorial } 5 \\
\end{array}$ & $\begin{array}{r}\text { Tutorial } 6 \\
\end{array}$ & \begin{tabular}{|l|} 
Tutorial 4 \\
\end{tabular} \\
\hline & Room: & Room: & Room: computer class \\
\hline & T5 & $\overline{T 6}$ & T4 \\
\hline $\begin{array}{l}14 \mathrm{~h} 00-14 \mathrm{~h} 20 \\
14 \mathrm{~h} 20-14 \mathrm{~h} 40 \\
14 \mathrm{~h} 40-15 \mathrm{~h} \\
15 \mathrm{~h} 00-15 \mathrm{~h} 20 \\
15 \mathrm{~h} 20-15 \mathrm{~h} 40\end{array}$ & $\begin{array}{l}\text { Security, electronic sig- } \\
\text { natures, pseudonym } \\
\text { services; Hospital in- } \\
\text { tranet development } \\
\text { De Meyer, Fiers } \\
\end{array}$ & Arvanitis & $\begin{array}{l}\text { Human Genetics } \\
\text { Bottu }\end{array}$ \\
\hline $15 \mathrm{~h} 40-16 \mathrm{~h}$ & Coffee & & \\
\hline \begin{tabular}{|l|}
$16 \mathrm{~h} 00-16 \mathrm{~h} 20$ \\
$16 \mathrm{~h} 20-16 \mathrm{~h} 40$ \\
$16 \mathrm{~h} 40-17 \mathrm{~h}$ \\
$17 \mathrm{~h} 00-17 \mathrm{~h} 20$ \\
\end{tabular} & & & \\
\hline $20 \mathrm{~h} 00-24 \mathrm{~h}$ & Conference Rec & eption & \\
\hline
\end{tabular}




\begin{tabular}{|c|c|c|c|}
\hline Mednet & 2000 & DAY 2 & Friday Nov. 24th. \\
\hline & Parallel 1 & Parallel 2 & Posters \\
\hline & Aud. 6 (Brouwer) & Aud. 2 (V. Driessche) & \\
\hline 9h00-9h15 & Opening session & & \\
\hline 9h15-10h15 & Keynote 1: Helga Rippen & & \\
\hline 10h15-10h30 & Discussion & & \\
\hline 10h30-11h & Coffee & & \\
\hline & & & \\
\hline & Oral 1: & Oral 2: & Poster 1: \\
\hline & Clinical applications 1 & Telemedicine 1 & \\
\hline $11 \mathrm{~h} 00-11 \mathrm{~h} 20$ & op06 & op59 & po64 \\
\hline $11 \mathrm{~h} 20-11 \mathrm{~h} 40$ & op22 & op05 & po86 \\
\hline $11 \mathrm{~h} 40-12 \mathrm{~h}$ & op44 & op12 & po87 \\
\hline $12 \mathrm{~h} 00-12 \mathrm{~h} 20$ & op29 & op41 & po11 \\
\hline $12 \mathrm{~h} 20-12 \mathrm{~h} 40$ & op30 & op49 & po54 \\
\hline $12 \mathrm{~h} 40-13 \mathrm{~h}$ & op76 & op53 & po26 \\
\hline $13 \mathrm{~h} 00-14 \mathrm{~h}$ & LUNCH & & \\
\hline $14 \mathrm{~h} 00-15 \mathrm{~h}$ & Keynote 2: Ilias lakovidis & & \\
\hline $15 \mathrm{~h} 00-15 \mathrm{~h} 20$ & Discussion & & \\
\hline & & & \\
\hline $15 \mathrm{~h} 20-15 \mathrm{~h} 40$ & Coffee & & \\
\hline & & & \\
\hline & Oral 3: & Oral 4: & Poster 2: \\
\hline & Medical education & Epidemiology/auth/publ. & \\
\hline $15 \mathrm{~h} 40-16 \mathrm{~h}$ & op09 & op04 & po45 \\
\hline 16h00-16h20 & op28 & op57 & po48 \\
\hline $16 \mathrm{~h} 20-16 \mathrm{~h} 40$ & op34 & op63 & po27 \\
\hline $16 \mathrm{~h} 40-17 \mathrm{~h}$ & op37 & op14 & po42 \\
\hline $17 \mathrm{~h} 00-17 \mathrm{~h} 20$ & op67 & op91 & po46 \\
\hline $17 \mathrm{~h} 20-17 \mathrm{~h} 40$ & op74 & op17 & po47, po68 \\
\hline 17h40-18h & op84 & op18 & po56, po83 \\
\hline & Evidence based medicine & Quality of information & \\
\hline $18 \mathrm{~h} 00-18 \mathrm{~h} 20$ & op95 & & po90 \\
\hline $18 \mathrm{~h} 20-18 \mathrm{~h} 40$ & op79 & MedCERTAIN workshop & po02 \\
\hline $18 \mathrm{~h} 40-19 \mathrm{~h} 00$ & op21 & & \\
\hline $20 h 00-24 h$ & Conference Dinne & & \\
\hline
\end{tabular}




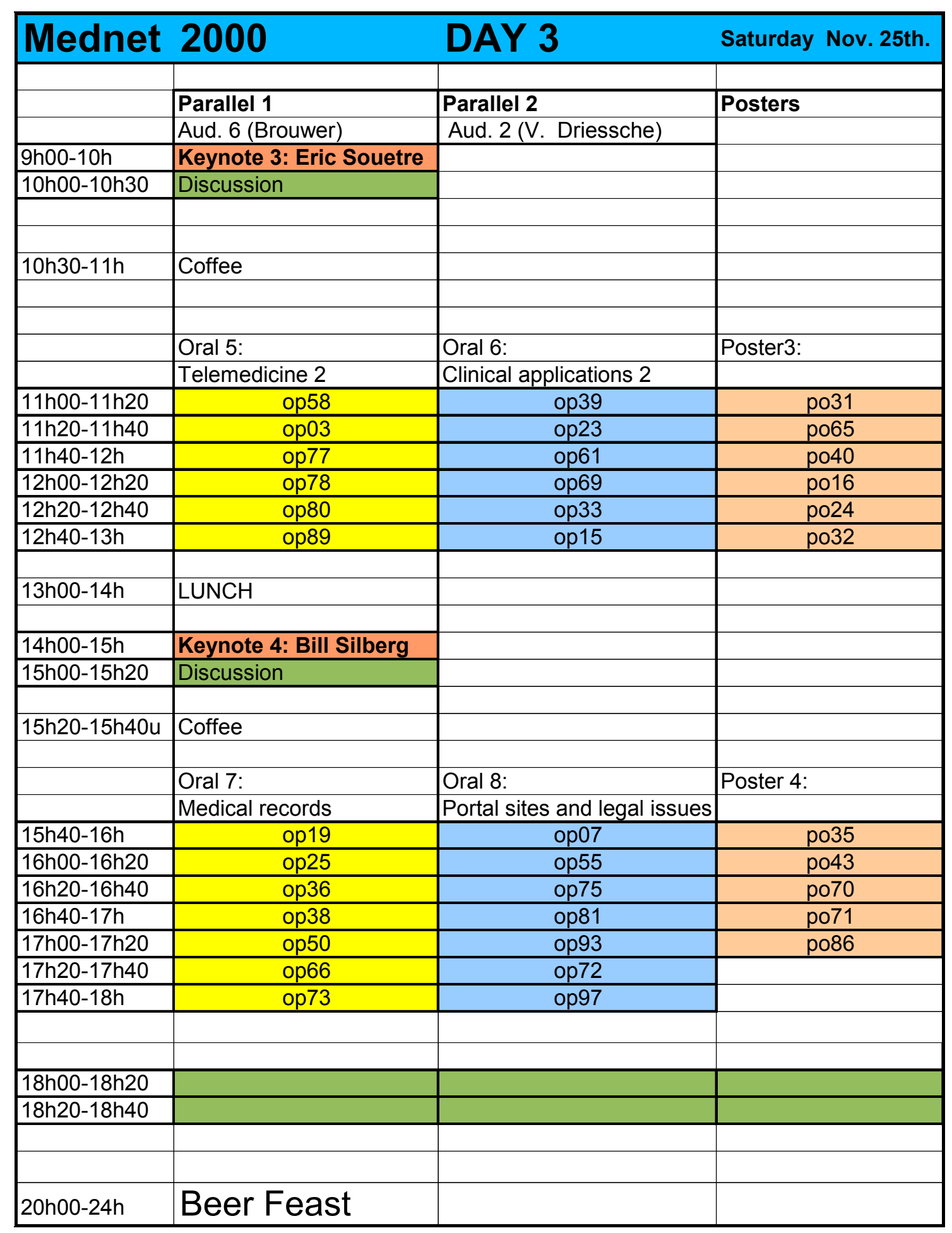




\begin{tabular}{|c|c|c|c|}
\hline \multicolumn{2}{|c|}{ Mednet 2000} & DAY 4 & Sunday Nov. 26th. \\
\hline 10h30-11h & Coffee & & \\
\hline & Tutorial 7 & Tutorial 8 & Tutorial 9 \\
\hline & Room: computer class & Aud. 6 & computer class 2 \\
\hline & T7 & $\overline{T 8}$ & T9 \\
\hline $11 \mathrm{~h} 00-11 \mathrm{~h} 20$ & PGP & Europortal & Web PACS \\
\hline$\frac{11 \mathrm{~h} 20-11 \mathrm{~h} 40}{11 \mathrm{~h} 40-12 \mathrm{~h}}$ & Putzeys, Michaux & Humphrey Gyde & $\begin{array}{l}\text { Jan Schillebeeckx } \\
\text { Erik Ranschaert }\end{array}$ \\
\hline $12 \mathrm{~h} 00-12 \mathrm{~h} 40$ & Coffee & & \\
\hline $12 \mathrm{~h} 40-13 \mathrm{~h}$ & & & \\
\hline $13 \mathrm{~h} 00-13 \mathrm{~h} 20$ & & & \\
\hline $13 \mathrm{~h} 20-13 \mathrm{~h} 40$ & & & \\
\hline $13 \mathrm{~h} 40-14 \mathrm{~h}$ & & & \\
\hline 13h00-14h20 & & & \\
\hline
\end{tabular}

